NASA Technical Memorandum 86003

NASA-TM-86003 19850009170

\title{
Satellite Stratospheric Aerosol Measurement Validation
}

\section{P.B. Russell and M.P. McCormick}

\section{LIBRARY ROPY \\ 12331565 \\ LANGLEY RESEARCH CENTER \\ LIBRARY, NASA \\ HAIIPTON, VIRGINIA}

\section{N/Sก \\ Natıonal Aeronautics and \\ Space Admınıstratıon}

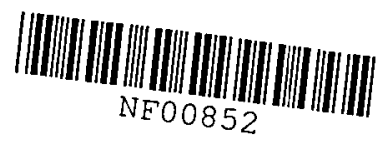


SATELLITE STRATOSPHERIC AEROSOL MEASUREMENT VALIDATION

\author{
P. B. Russell* and M. P. McCormick**
}

*NaSA Ames Research Center, Moffett Fleld, CA 94035, U.S.A. **NASA Langley Research Center, Hampton, VA 23665, U.S.A.

\title{
ABSTRACT
}

The validity of the stratospheric aerosol measurements made by the satellite sensors SAM II and SAGE has been tested by comparing their results with each other and with results obtained by other techniques (11dar, dustsonde, f1lter, impactor). The latter type of comparison has required the development of special techniques that (1) convert the quantity measured by the correlative sensor (e.g. particle backscatter, number, or mass) to that measured by the satellite sensor (extinction), and (2) quantitatively estimate the uncertalnty in the conversion process. The results of both types of comparisons show agreement within the measurement and conversion uncertainties. Moreover, the satellite uncertalnty is small compared to aerosol natural varlability (caused by seasonal changes, volcanoes, sudden warmings, vortex structure, etc.). Hence, we conclude that the satellite measurements are valid.

\section{INTRODUCTION}

The satellite sensors Stratospheric Aerosol Measurement (SAM II) and Stratospheric Aerosol and Gas Experiment (SAGE) have been developing a global data base on stratospheric aerosol behavior since they were launched in October 1978 and February 1979 , respectively $/ 1-6 /$. To rest the validity of this data base, an extensive program of comparisons has been conducted, both between the two satellite sensors and between other sensors and the satellite sensors.

\section{COMPARISONS BETWEEN SAM II AND SAGE}

As described in more detall by Yue et al. $17 /$, the SAM II measurements are confined to the two lat1cude bands: $64^{\circ}-80^{\circ} \mathrm{N}$ and $64^{\circ}-80^{\circ} \mathrm{S}$, whereas the SAGE measurements range from $72^{\circ} \mathrm{S}-$ to $72^{\circ} \mathrm{N}$. Thus, there are occasions when the measurements nearly overlap, permitting comparisons.

Although SAM II and SAGE use the same measurement princtple (sun photometry through the Earth's atmospheric 11mb, ylelding vertical profiles of aerosol extinction), there are significant differences between the instruments used and data-reduction procedures. For example, the sensors fly on separate satellites in different orbits, leading to considerably different viewing geometries and sampling durations even when the sampled air volumes are adjacent. Also, the SACE Instrument uses a holographlc grating to disperse the sunlight, while the SAM II instrument uses an interference filter for spectral discrimination. SAGE measurements are quantized to 12 bits accuracy ( 1 part in 4096), while SAM II measurements are quantized to 10 bits accuracy ( 1 part in 1024). The higher quantization error for SAM II measurements is apparent when the measured aerosol extinction levels are low.

The handing and processing of the data from each instrument are also different. The telemetry and raw data processing for the two satellite systems are separately operated under different organizations at NASA's Goddard Space Flight Center. In addition, the data reduction schemes for the two experiments are considerably different. For example, the geometric height determination for the SAM II-Inferred vertical profiles is based primarily on the refracted sunshape fitting scheme $/ 8 /$. For the SAGE data, it is determined by matching the measured slant-path optical depth profiles at the short wavelength channel to a computed profile from the given vertical temperature and pressure profiles. The inversion algorithms for the two instruments are also different. SAGE inversion uses a nonlinear Iterative Invergion algorithm where all the four wavelength data are cyclically updated, while the SAM II 1nversion algorithm is basically a linear inverse matrix-type inversion applied to the single wavelength measurement. The comparisons between SAM II and SAGE 1.0-um wavelength inverted data, therefore, can provide a critical assessment of 
the end-to-end performance of the two measurement systems, and can validate the consistency of the inferred aerosol vertical extinction profiles.

By the end of 1979 there were five occasions when the latitude coverages of SAM II and SAGE overlapped. We will show representative comparisons, both between individual profiles and between helght-vs.-longltude cross sections generated by combining many profiles.

SAM II and SAGE Individual Profile Comparisons

F1gure 1(a) shows a typical SAM II/SAGE compartson for a case of hackground aerosol concentrations, where the measurement coordinates differed by $0.26^{\circ}$ latitude, $0.86^{\circ}$ Iongitude, and 0.06 hours. (The coordinates give the location and t1me when each satellite's Sun-viewing 1 imb path was tangene at $20 \mathrm{~km}$ alt1tude.) The error bars shown are \pm 10 , derived from the error analysis of Chu and MeCormick $/ 8 /$. They include uncertaincies in pointing, radiometric impreciston, digicizer truncation, and uncertainties in the molecular density profile at the measurement location. The profiles are typical in that they show a steep decrease of aerosol extinction near the tropopause height (Indicated by the arrow), followed by a region of nearly constant extinction extending from about $2 \mathrm{~km}$ above the tropopause to about $8 \mathrm{~km}$, followed by a relatively steep drop-off.

As can be seen, within the stratosphere the SAM II and SAGE profiles agree to within error bars. Below the tropopause, differences often exceed error bars. We attribute this behavior (which is typical of other proftle comparisons) to the patchiness of the tropospheric aerosol structure, coupled with the fact that SAM II and SAGE tropospheric limb paths can be separated by considerably more than the differences stated above and shown in Figure 1. (Recall that those differences apply to the 20-km tangent height.) In cases where tropospheric clouds are present, SAM II/SAGE differences can grearly exceed those shown in Figure 1(a) because of the large inhomogeneities of clouds. For other examples and further discussion, see $/ 7 /$.

Figure I(b) shows a SAM II/SAGE comparison for a case of unusual background stratospheric aerosol structure. Note that, in this case, particulate exinction decreases rapidly from about $2 \times 10^{-4} \mathrm{~km}^{-1}$ at $11 \mathrm{~km}$ ale1tude to about $2 \times 10^{-5}$ at $18 \mathrm{~km}$ altleude, racher than staying nearly constant which is far more typical behavior in this height region (cf. Figure $1(a)$ ). This inceresting phenomenon was not a single example but continued for several days. The mechanism behind this phenomenon is still under Investigation /6/; however, $1 t$ is possible that this is the result of a strong polar vortex and is typical of the air mass inside the vortex experiencing a subsidence and sedimentation. The important point here is that SAM II and SAGE are in excellent agreement in documenting this atmospheric behavior.
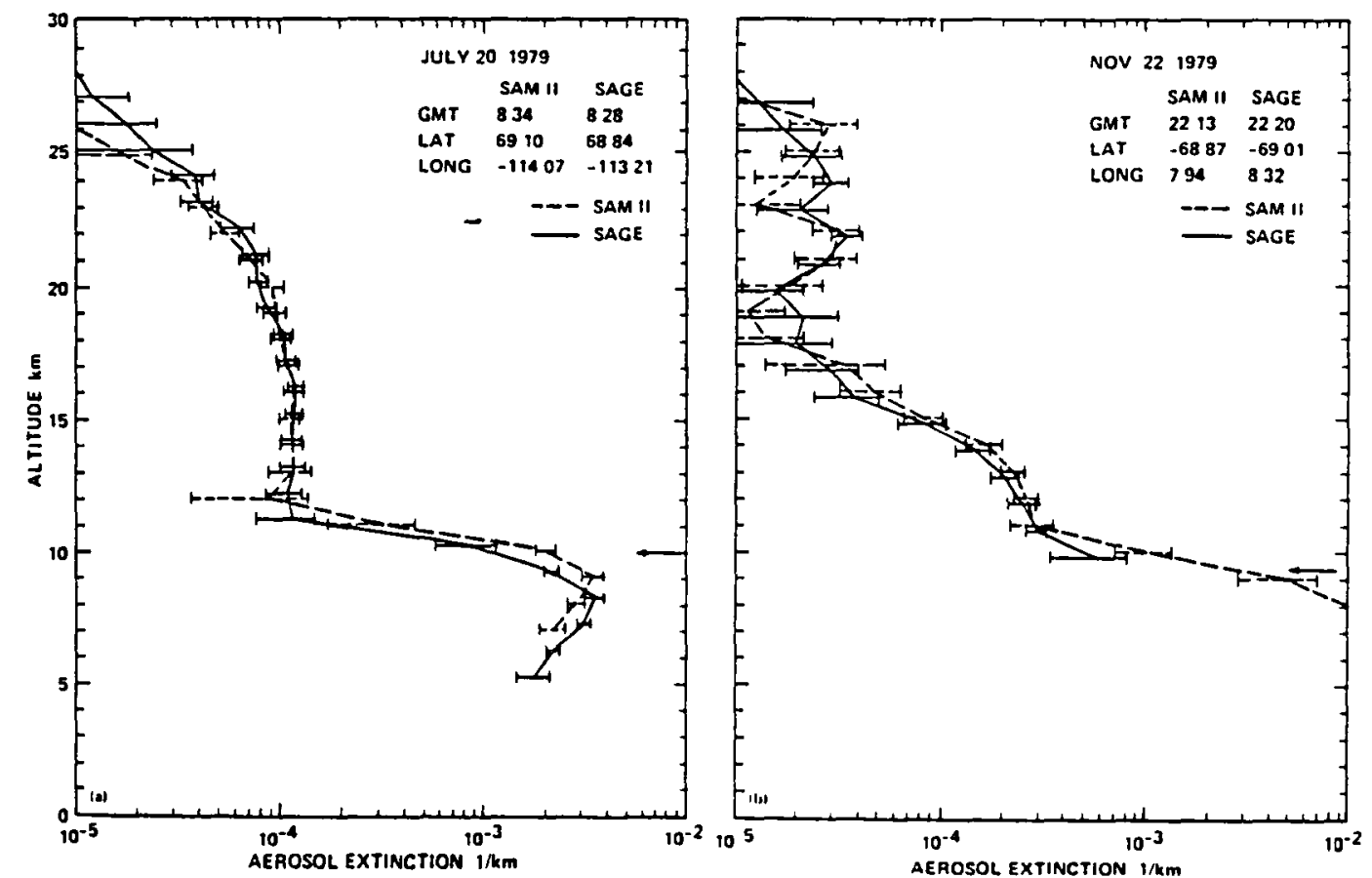

Fig. 1. Selected comparisons between SAM II and SAGE aerosol extinction profile measurements. 


\section{SAM II and SAGE Concour P1or Comparisons}

Figure 2 shows two examples from SAM II and SAGE in which height vs. longitude cross sections are compared. For each sensor, these cross sections are generated by inputting to a contour plotting routine all the vertical profiles measured in a single day. Numbers shown on the contours are in units of $10^{-5} \mathrm{~km}^{-1}$ and are separated by multiples of $10^{0.2}$ (about 1.58). As In the individual profiles shown in Figure 1, only values of extinction greater than $10^{-5} \mathrm{~km}^{-1}$ are shown; the solld IInes represent data obtained by SAGE, and the dashed lines are for SAM II. The longttudinal positions where SAGE and Sam II data were caken are ind1cated by tick marks at the top and bottom of each flgure, respectively. The latitudinal coverage for each sensor for that day is also listed. The positions of each measurement indicated in Figures 2(a) and (b) are those corresponding to a $11 \mathrm{mb}$ path at $20 \mathrm{~km}$ altitude. On a given day, 15 is about the maximum number of sunrise or sunset measurement opportunities for each sensor.

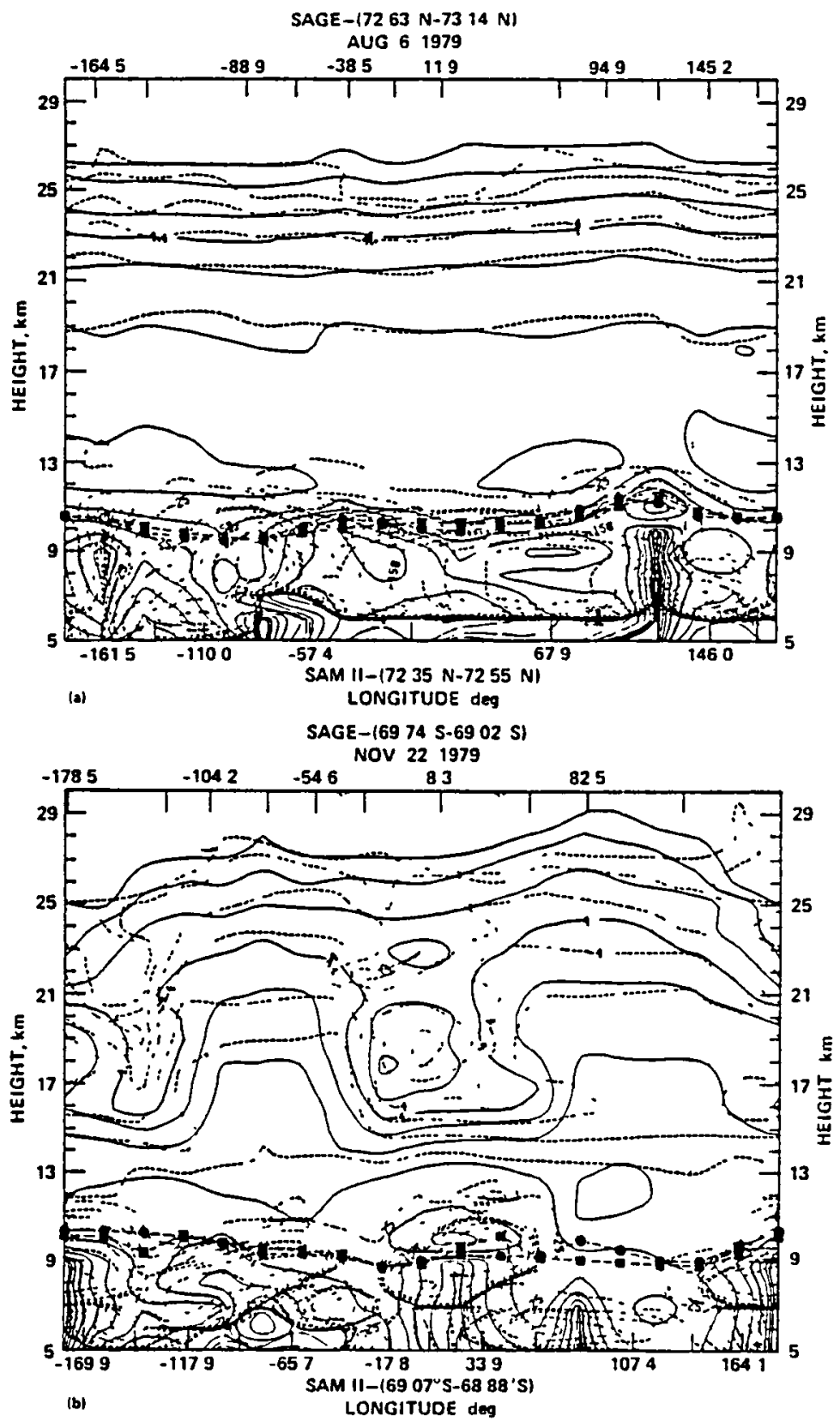

F1g. 2. Selected comparisons between helght/longitude cross sections of extinction derived from SAM II and SAGE measurements. Solid lines: SAGE; dashed: SAM II. 
The reader should be cautioned that computer-generated contours such as those in Figure 2 can lead to apparent differences and possible misinterpretations, because profiles at different longitudes are used in their lndependent construction. Many elmes and for various reasons, a profile is missing; thus, instead of a $24^{\circ}$ (SAGE) or $26^{\circ}$ (SAM II) separation between profiles for interpolation, twice this longitude interval is used. Sometimes two consecutive profiles are missing, causing an even larger interpolation distance. If, for example, there is a localized enhancement in the SAM II data that colncides with a data-vold area for SAGE, a very different contour would be constructed for the two sensors.

From the plotted contours in Figure 2(a) which represents a very homogeneous condition, it can be seen that there is very good agreement between the SAM II and SAGE measurements for the altitude region more than $2 \mathrm{~km}$ above the tropopause height (1ndicated by the c1rcles for SAGE data and squares for SAM II data). Altitude differences are in general less than $1 \mathrm{~km}$ between contours of the same aerosol extinction, but obtained from different sensors. As mentioned earlier, the small differences between SAM II and SAGE measurements of aerosol extinction in the region around the tropopause may be due to vartable tropopause heights, and the varlable and patchy clouds associated with them. The small differences in the region above $24 \mathrm{~km}$ are caused by relatively large systematic errors associated with data in that region. Even though there are small differences between SAM II and SAGE results in those two regions, they are still in good agreement within their respective uncertainties. Most of the differences seen In Figure $2(a)$ are due to data volds and their effects on the contour plote1ng.

The aerosol extinction contour plots shown in Figure 2(b) represent the case in which there are two regions where aerosol extinction is very different. As described previously, this phenomenon is associated with a well-established polar vortex /6/. Figure 2(b) vividly shows the distortion generated by having data gaps at a particular longleude. Even here, however, both contours show the major features of the stratospheric aerosol on that day.

\section{COMPARISONS OF OTHER SENSORS WITH SAM II AND SACE}

Of all the nonsatelltte aerosol sensors and samplers available to validate SAM II and SAGE (e.g. 11dars, optical particle councers, filter collectors, and various cypes of 1mpactors), none measures extinction, which is the quantity measured by SAM II and SAGE. Instead, the nonsatellite sensors measure such quantitles as particle backscatter, number, and mass. Thus, before using these correlative sensors to validate the satellite sensors, procedures must be developed that (1) convert the correlative-measured property to the satellitemeasured one, and (2) quantitatively estimate the uncertainty in the conversion process.

\section{Conversion Procedures and Uncercalnties}

It 15 well known that there are no unlque factors (or conversion ratios) relating extinction to mass, number, or backscatter for the stratospheric aerosol because the factors (ratios) depend on particle size diseribution, composition, and shape-all properties which are known to vary. Nevertheless, one can determine the range of these properties by using concurrent measurements, physical principles, and climatological information. Having defined the ranges of size distribution, composition, and shape, one can then define the corresponding ranges of possible conversion ratios. These ranges may be expressed as mean values and uncertainties, each as functione of height.

Figure 3 1liustrates schematically our technique for accomplishing and automating the above process. The technique is explained in deta11 in /9-11/. In brief, it consists of the following steps:

1. Define the range of aerosol refraction indices expected in specified helght regions.

2. Parameterize a wide range of model relative-size distributions in terms of the "channel rat10," N0.1s/N0.25, where $N_{x}$ is the number of particles with radil greater than $x \mu m$. (We chose channel ratio as the key size-distribution parameter because it is measured by dustsondes in most of our correlative experiments, and when measurements are not avallable, there is a large data base from which channel ratio can be estimated. Moreover, for the stratospheric aerosol, knowledge of the channel ratio provides a very useful constraint on conversion ratio values.)

3. Perform Mie-scattering calculations for a very large mumber of combinations of refractive Indices and stze distributions, and organize the results to give mean and standard deviation curves of conversion ratios (e.g. extinction-to-mass) as a function of $\mathrm{N}_{0.25} / \mathrm{N}_{0} .25$ (see $19,10 /$ for examples). The mean curves give the most probable converston ratio for a particular $N_{0.1 s} / N_{0} .25$ value, and the standard deviations give the $1 \sigma$ uncertainty (1.e. expected rms error) that results from imperfect knowledge of refractive Index and size-distribution details (see the example in Figure $3(a)$ ). 


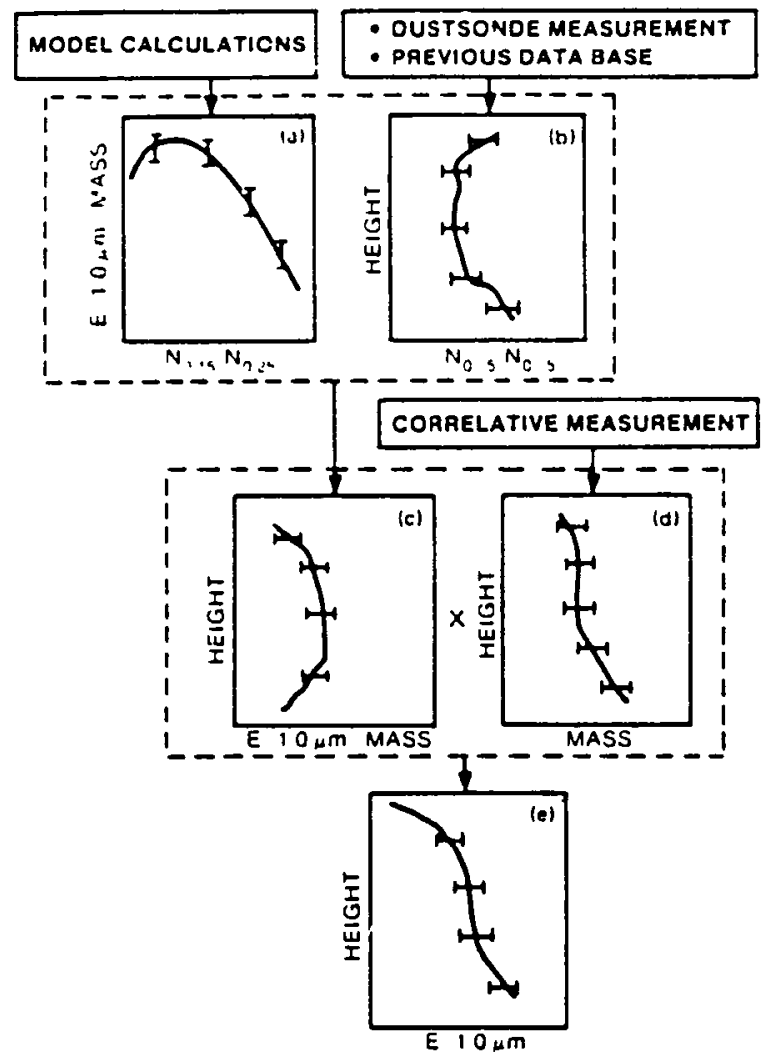

F1g. 3. Schematic of the procedure used to convert measurements of correlative properties (e.g., mass, number, backscatter) to estimates of extinction.

4. Combline curves of conversion ratio versus $\mathrm{N}_{0.15} / \mathrm{N}_{0.25}$ with measured (or estimated) vert1cal profiles of $\mathrm{N}_{0.15} / \mathrm{N}_{0.25}$ (Flgure $3(\mathrm{~b})$ ) to obtain vertical profiles of conversion ratio and its uncertainty (Figure $3(\mathrm{c})$ ).

5. Multiply measured profiles of the correlative property (number, backscatter, mass; e.g. Figure 3(d)) by the corresponding profile of conversion ratio (extinction-to-number, extinction-to-mass, or extinction-to-backscatter), and combine measurement and conversion uncertainties, using conventional error-propagation methods (see /9-10/). The result is the correlative extinction profile exemplified by Figure $3(e)$.

Resules: Lidar vs. SAM II; Voleanic Aerosol

Figure 4 shows two examples of comparisons between extinction profiles measured by SAM II and those estimated from airborne 11dar measurements of backscatter. In both cases, the stratospheric aerosol concentration is greatly enhanced over the background levels of Figures 1 and 2, primarily because of the EI Chfchon volcanic infection. Due to this volcanic perturbation, the conversion procedure described above and in Figure 3 was modified slightly, both because (1) simultaneous dustsonde measurements of $N_{0} .15 / N_{0} .25$ were not available (and no "typical El Chichon" $N_{0}$ is $/ \mathrm{N}_{0.25}$ proffles could be estimated from the sparse data available elsewhere), and (2) the preponderance of large particles in the El Chichon cloud decreases the usefulness of $N_{0.15} / N_{0.2 s}$ in constralning size distribution. Instead of relying exclusively on $\mathrm{N}_{0.15} / \mathrm{N}_{0.25}$, 6-channel dustsonde measurements of post-El Chichon size distributions were used to define mean and standard-deviation extinction-to-backscatter ratios. These were then combined with the lidar-measured backscatter profiles to yfeld the dashed extinction curves and error bars in Figure 4. It can be seen that, in spite of the uncertaincy in particle size distribution, the resulting uncertainty in lidar-1nferred extinction is relatively small ( $₫ 20 \%$ in the aerosol peak). Within this uncertainty, there is a very good agreement between the satellite-measured and lidar-inferred extinction profiles.

Results: Lidar and Dusesonde vs. SAM II, Background Aerosol

Figure 5 shows results from a validation experiment $/ 10 /$ conducted at Sondrestrom, Greenland, in November 1978, during "background" (nonvolcanic) aerosol conditions. In this case, both a lidar and a dustsonde particlpated, and the conversion procedure shown in Figure 3 was 

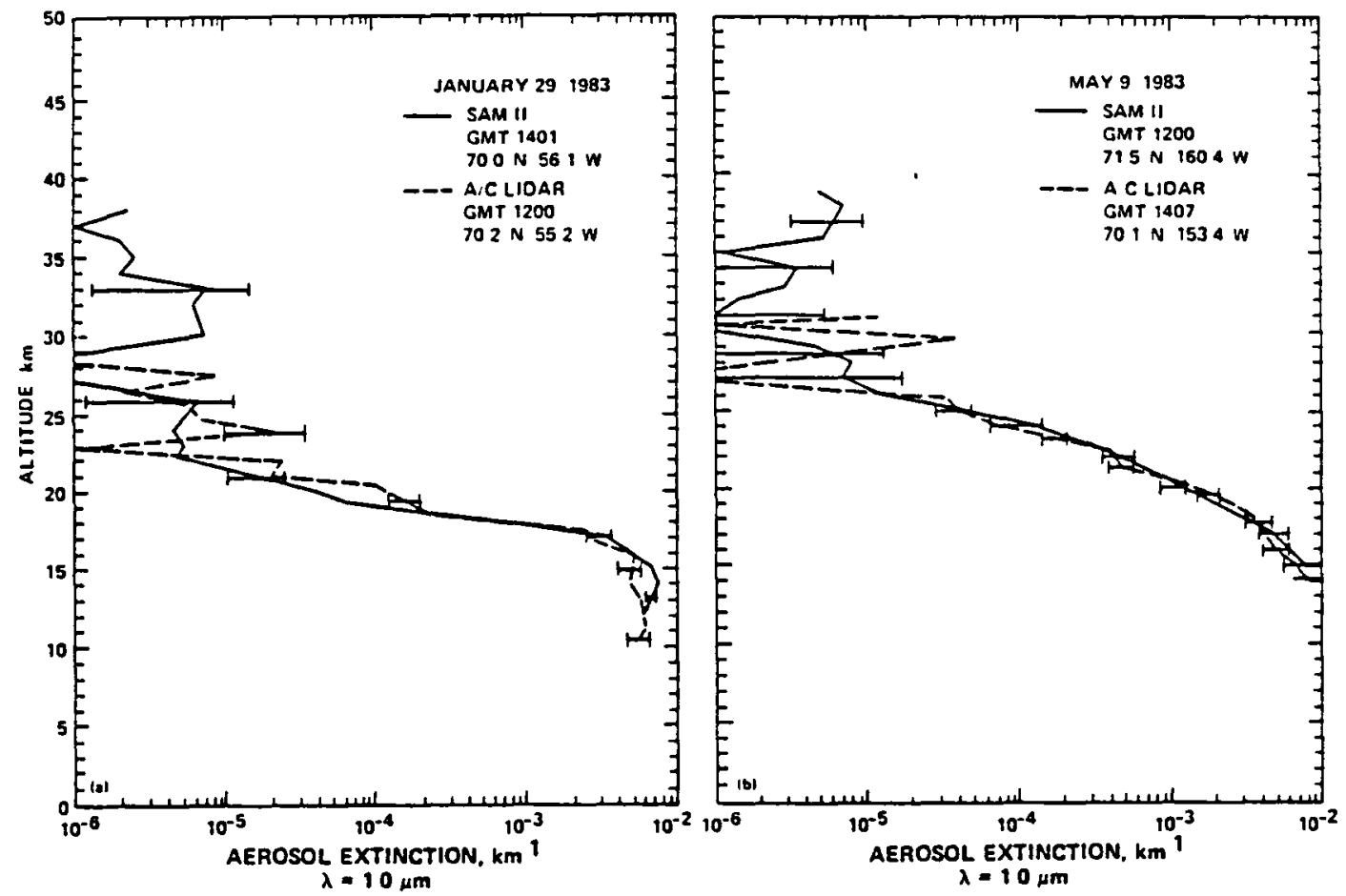

Fig. 4. Selected comparisons between extinction profiles measured by SAM II and derived from alrborne lidar measurements.

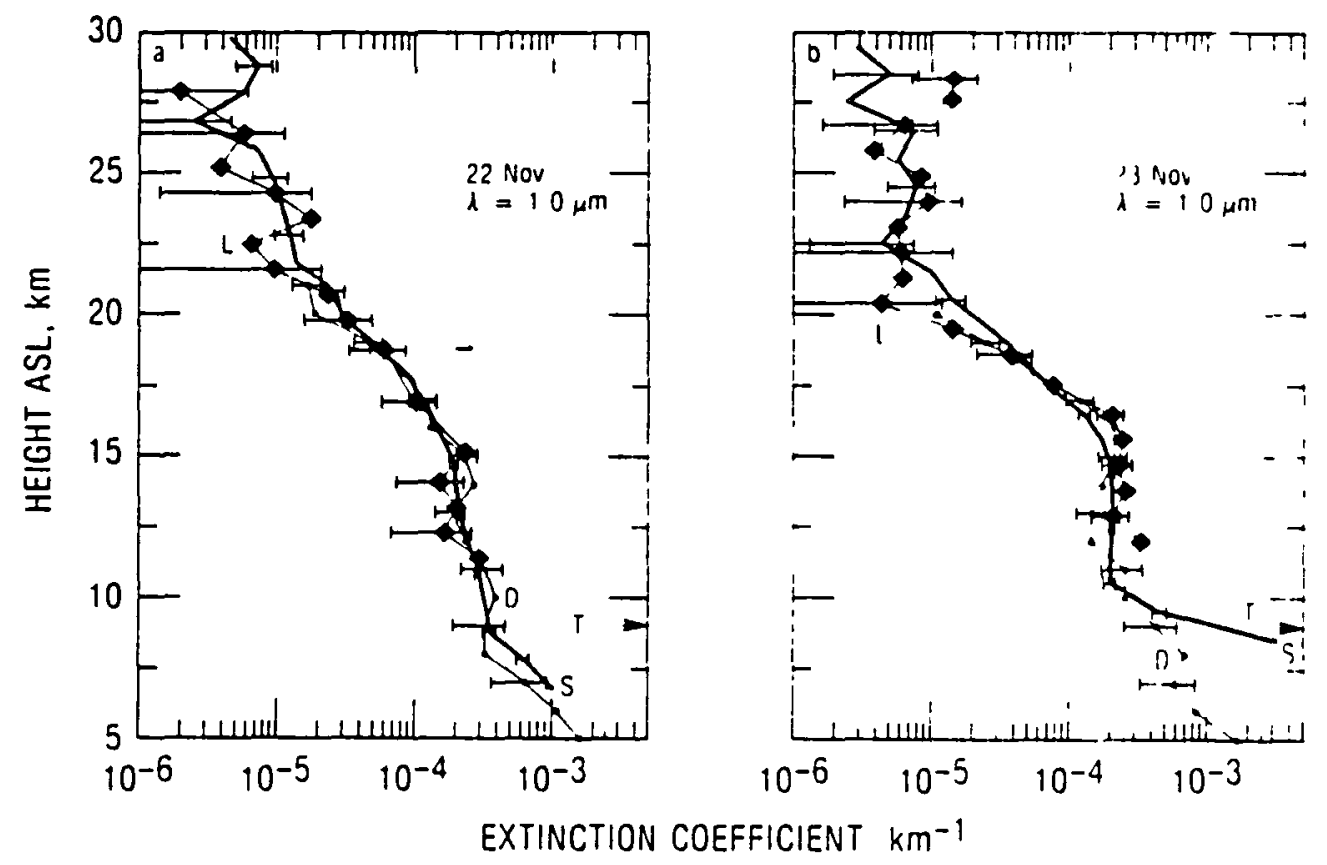

F1g. 5. Comparison between extinction profiles measured by SAM II (S) and derived from lidar (L) and dustsonde (D) measurements, November 1978, Sondrestrom, Greenland. 
followed exactly. The error bars on the 11dar- and dustsonde-1nferted extinction curves give the comblned result of measuremenc and conversion uncertainty. (For the dustsonde, conversion errors dominate the total, for the 1 idar, measurement errors dominate. See $/ 10 /$ for detalls.) For both days $(22,23$ Nov.) at all helghts above the tropopause, the extinction profiles measured by SAM II agree with those inferred from the lidar and dustsonde data to within the respective measurement and conversion uncertainties. Below the tropopause on 23 Nov. 1978 (FIgure 5), the SAM II-measured extinction exceeds that measured by the dustsonde. We belleve this is caused by the SAM II limb path intercepting patchy clrrus clouds which were not efficiently sampled by the dustsonde.

Results: Lidar, Dustsonde, F1lter, and Impactor vs. SAM II and SAGE. Background Aerosol

Figure 6 shows the results from a validation experiment /11,12/ conducted at Poker Flat, Alaska in July 1979, also during background aerosol conditions. The procedure fllustrated In Figure 3 was used to derive extinction profiles and uncertainties from the 11dar, dustsonde, and filter measurements of backscatter, number, and mass, respectively. We have also derived extinction values from the size distributions measured by wire impactor. However, our error analysis for these values differs from that for the other sensors because of the more detailed nature of the wire-1mpactor data. Specifically, the wire impactor measures differential particle number densities $\left(d N_{r} / d r\right)$ at a large number $(-15)$ of radius intervals, Instead of directly measuring integral properties (such as $\mathrm{N}_{0.15}, \mathrm{~N}_{0.25}$, mass, and backscatter). Thus, the wire impactor ylelds detalled size-distribution information, but each $\mathrm{dN}_{\mathrm{r}} / \mathrm{dr}$ data point has assoctated uncertainties, amounting to $\pm 8 \%$ to \pm 207 in $r$ and highly variable amounts in $\mathrm{dN}_{r}$. Because of the steepness of background stratospherfc particle-sizt distributions, the uncertaintes in $r$ lead to considerably larger uncertainties in some wire-impactor-derived integral properties (such as $\mathrm{N}_{0.15} / \mathrm{N}_{0} .2 \mathrm{~s}$, mass, and extinction).

A complete analysis of the $1 \sigma$ uncertalnties in these impactor-derfved integral properties is very complicated; however, to lllustrate the magnitudes possible, we show in Figure 6 two wire-impactor-inferred extinction values at each measurement height. One value is derived from the "best-estimate" 1mpactor-inferred size distribution, and the other is derived from this same distribution with all radil reduced by $15 \%$. Note that this small radius shift (which is within the wire-impactor measurement uncertainty-95z confidence 11mits), reduces extinction by a factor of 2 to 3 . Thus, the more detalled wire-fmpactor data, when used as an indirect means to estimace extinction (and certaln other integral properties), yield relatively large uncertainties.

Figure 6 also shows the profiles of extinction measured by SAM II and SAGE. To within the associaced measurement and conversion uncertainties, the SAM II and SAGE profiles agree with each ocher and with the values derived from the simultaneous dustsonde, filcer, wireimpactor, and lidar measurements.
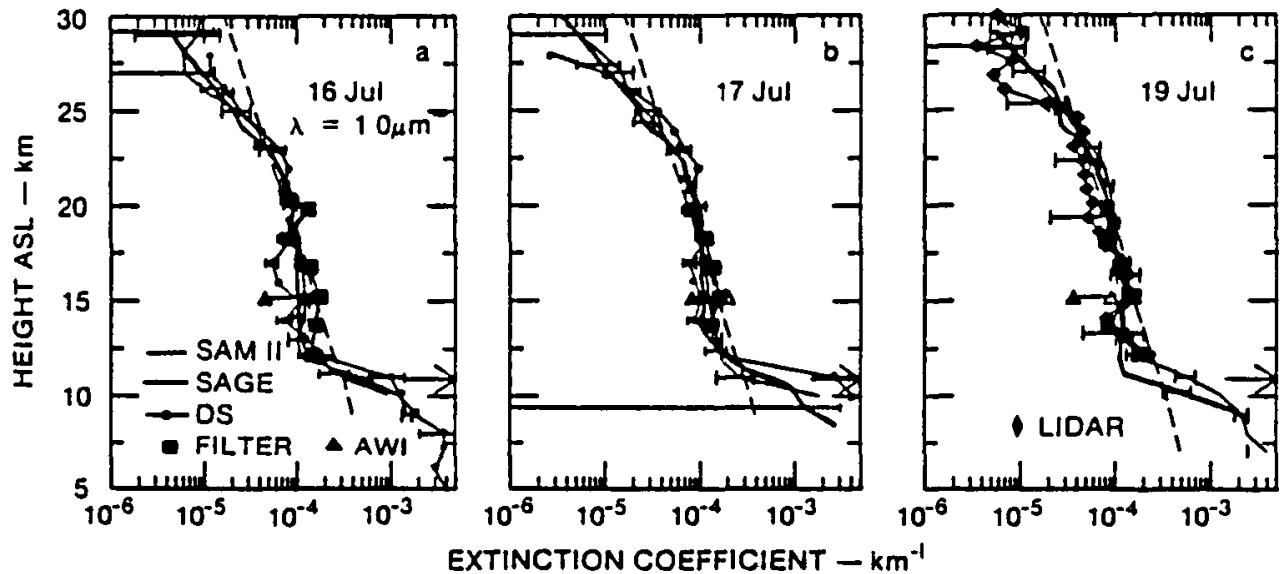

Fig. 6. Comparison of extinction profiles measured by SAM II and SAGE with those derived from dusesonde (DS), f1lter, 11dar, and Ames wire-impactor (AWI) measurements. For each hortzontal pair of AWI data points, the point on the left results from reducting all particle radil by $15 z$. 


\section{CONCLUSIONS}

The SAM II and SAGE measurements agree with each other and with values inferred by other techniques to within the respective measurement and conversion uncertaincies. Moreover, the satellite measurement uncertaincy is small compared to aerosol natural varlability. Hence, we conclude that the sacellite measurements are val1d.

\section{ACXNOWLEDGMENTS}

The SAM II and SAGE correlative experiments were made possible through the generous cooperation of Individuals and agencies too numerous to mention here. However, we are particularly grateful to J. Rosen, D. Hofmann, W. Fuller, N. Farlow, G. Ferry, V. Oberbeck, B. Gandrud, and A. Lazrus for supplying data presented in this paper, to L. R. McMaster for overall coordination of the experiments, and to $G$. Yue for data analyses.

\section{REFERENCES}

1. M.P. McCormlck, P. Hamill, T.J. Pep1n, W.P. Chu, T.J. Swlssler, and L.R. McMaster, Satellite studies of the stratospheric aerosol, Bull. Am. Meteor. Soc. 60, 1038 (1979).

2. M.P. McCormick, Aerosol measurements from Earth orbit1ng spacecraft, Adv. Space Res. 2, 73 (1982).

3. M.P. McCormick, W.P. Chu, G.W. Grams, P. Hamill, B.M. Herman, L.R. McMaster, T.J. Pepin, P.B. Russel1, H.M. Steele, and T.J. Swissler, HIgh-latitude stratospheric aerosols measured by the SAM II satellice system in 1978 and 1979 , Sclence 214, 328 (1981).

4. M.P. MeCormick, G.S. Kent, G.K. Yue, and D.M. Cunnold, Stratospheric aerosol effects from Soufriere, St. Vincent as measured by the SAGE satellite system, Science 216, 1115 (1982a).

5. M.P. McCormick, H.M. Steele, P. Hamill, W.P. Chu, and T.J. Swissler, Polar stratospheric cloud sightings by SAM II, J.Atmos. Sc1. 39, 1387 (1982b).

6. M.P. McCormick, C. Trepte, and G.S. Kent, Spatial changes in the stratospheric aerosol assoclated with north polar vortex, Geophys. Res. Lett. 10, 941 (1983).

7. G.K. Yue, M.P. McCormick, and W.P. Chu, A comparative seudy of aerosol extinction measurements made by the SAM II and SAGE satellite experiments, J. Geophys. Res., in press (1984).

8. W.P. Chu and M.P. McCormick, Inversion of stratospheric aerosol and gaseous constitutents from spacecraft solar extinction data in the 0.38-1.0 $\mathrm{mm}$ wavelength regton, Appl. Optics 18,1404 (1979).

9. P.B. Russell, W. Viezee, R.D. Hake, W.P. Chu, J.M. Livingston, and T.J. Pepin, Satellite and correlative measurements of the stratospheric aerosol. I: An optical model for data conversions, J. Atmos. Sc1. 38, 1279 (1981a).

10. P.B. Russel1, M.P. McCormick, T.J. Swissler, W.P. Chu, J.M. Livingstone, W.H. Fuller, J.M. Rosen, D.J. Hofmann, L.R. McMaster, D.C. Woods, and T.J. Pep1n, Satell1te and correlative measurements of the stratospheric aerosol: II. Comparison of measurements made by SAM II, dustsondes, and an airborne 11dar, J. Atmos. Sci. 38, 1295 (1981b).

11. P.B. Russe11, M.P. McCormick, T.J. Swissler, J.M. Rosen, D.J. Hofmann, and L.R. McMaster, Satellite and correlative measurements of the stratospheric aerosol. III. Comparison of measurements by SAM II, SAGE, dustsondes, filters, impactors, and lidar, J. Atmos. Sc1. 41, in press (1984).

12. N.H. Farlow, V.R. Oberbeck, D.S. Colburn, G.V. Ferry, H.Y. Lem, and D.M. Hayes, Compar1son of stratospher1c aerosol measurements over Poker Flat, Alaska, July 1979, Geophys. Res. Lett. 8, 15 (1981). 


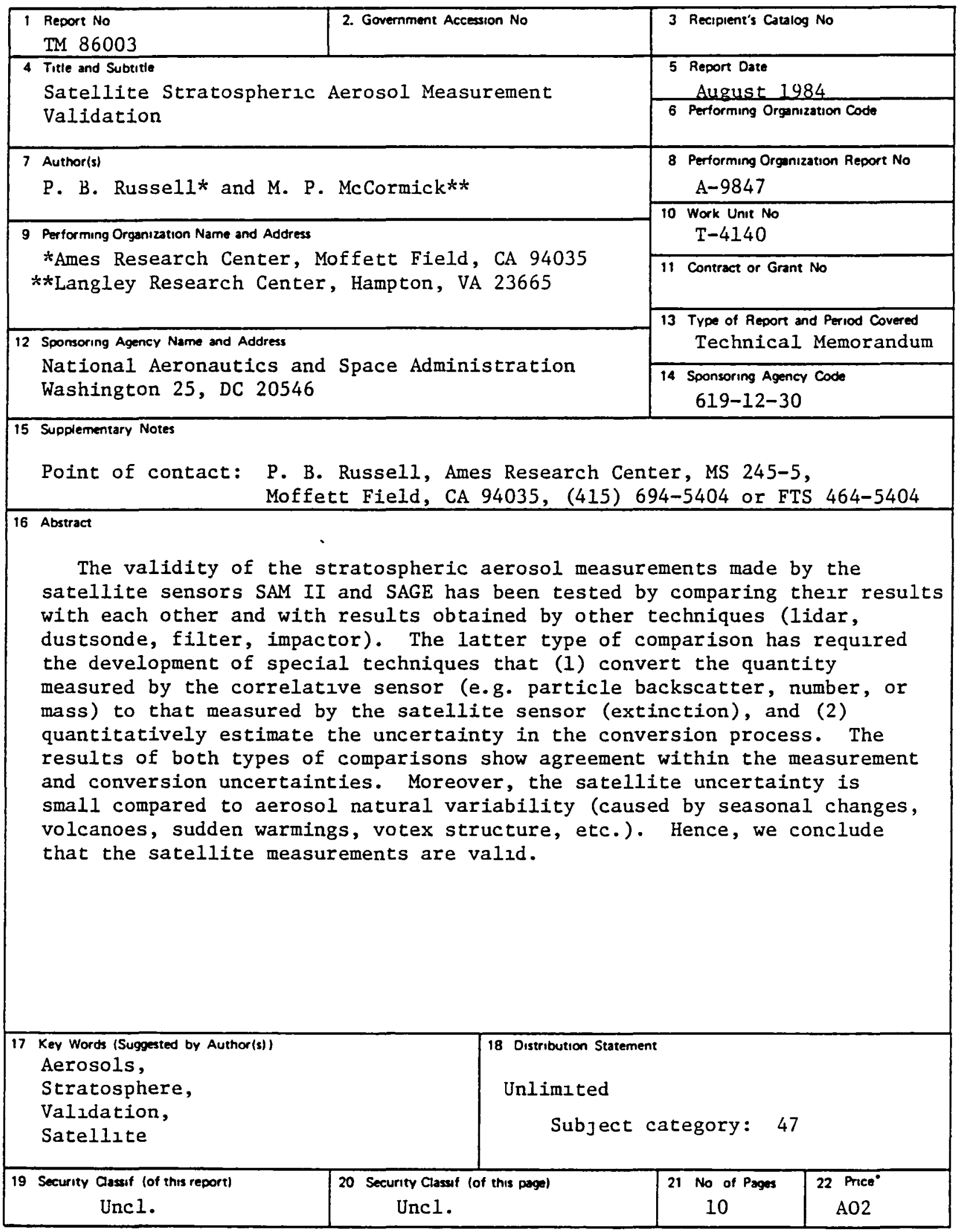


End of Document 